\title{
Detection of colorectal polyps in humans using an intravenously administered fluorescent peptide targeted against c-Met
}

\author{
Jacobus Burggraaf ${ }^{1}$, Ingrid M C Kamerling ${ }^{1}$, Paul B Gordon ${ }^{2}$, Lenneke Schrier ${ }^{1}$, Marieke L de Kam ${ }^{1}$, \\ Andrea J Kales ${ }^{1}$, Ragnar Bendiksen ${ }^{2}$, Bård Indrevoll ${ }^{2}$, Roger M Bjerke ${ }^{2}$, Siver A Moestue ${ }^{2}$, Siavash Yazdanfar ${ }^{3}$, \\ Alexandra M J Langers ${ }^{4}$, Marit Swaerd-Nordmo ${ }^{2}$, Geir Torheim ${ }^{2}$, Madhuri V Warren ${ }^{5}$, Hans Morreau ${ }^{6}$, \\ Philip W Voorneveld ${ }^{4}$, Tessa Buckle ${ }^{7}$, Fijs W B van Leeuwen ${ }^{7}$, Liv-Ingrid Ødegårdstuen ${ }^{2}$, Grethe T Dalsgaard ${ }^{8}$, \\ Andrew Healey ${ }^{2} \&$ James C H Hardwick ${ }^{4}$
}

\begin{abstract}
Colon cancer prevention currently relies on colonoscopy using white light to detect and remove polyps, but small and flat polyps are difficult to detect and frequently missed when using this technique. Fluorescence colonoscopy combined with a fluorescent probe specific for a polyp biomarker may improve polyp detection. Here we describe GE-137, a water-soluble probe consisting of a 26 -amino acid cyclic peptide that binds the human tyrosine kinase c-Met conjugated to a fluorescent cyanine dye. Intravenous administration of GE-137 leads to its accumulation specifically in c-Met-expressing tumors in mice, and it is safe and well tolerated in humans. Fluorescence colonoscopy in patients receiving intravenous GE-137 enabled visualization of all neoplastic polyps that were visible with white light (38), as well as an additional nine polyps that were not visible with white light. This first-in-human pilot study shows that molecular imaging using an intravenous fluorescent agent specific for c-Met is feasible and safe, and that it may enable the detection of polyps missed by other techniques.
\end{abstract}

Colorectal cancer (CRC) is a major cause of cancer death ${ }^{1}$, and colonoscopy is firmly established as the mainstay of CRC prevention. Evidence that CRC can be prevented by the removal of polyps is strong, especially for cancers of the left colon ${ }^{2}$. However, colonoscopy using current techniques only provides partial protection overall and limited, if any, protection in the right colon ${ }^{3,4}$. Although the aggressive biology of some cancers may in part explain this lack of complete protection, it is likely that limitations in polyp detection using current techniques are primarily responsible ${ }^{5}$.

Technical aspects of endoscopic imaging have a major role in determining polyp detection rates, together with human factors such as the quality of bowel preparation and the skill of the endoscopist ${ }^{6}$. Imaging at colonoscopy is currently performed using white light (WL), and polyps are detected by operators who are trained to discriminate polyps from normal colon by recognizing characteristics such as protrusion into the lumen and mucosal color changes. However, these features are less discriminatory in smaller and non-polypoid lesions, leading to miss rates of up to $25 \%$ (ref. 7). Combining targeted molecular probes and advanced imaging technology could improve polyp detection. Several biomarkers and detection systems have shown promise in preclinical trials ${ }^{8,9}$, but only topically applied agents have thus far been tested in humans ${ }^{10,11}$. These agents suffer from the major disadvantage that application to the whole surface area of the colon is seldom achievable.

c-Met overexpression has been shown to occur as an early event in the colorectal adenoma-carcinoma sequence ${ }^{12}$ making it a suitable biomarker for colorectal neoplasia ${ }^{13}$. In addition, its expression on the cell membrane makes extracellular epitopes accessible for targeting with fluorescent imaging agents. Here we report our initial experiences with fluorescence-guided colonoscopy using the imaging agent GE-137 (European Clinical Trials Database registration number 2010-019197-33; publicly accessible via the CCMO register (https:// www.toetsingonline.nl/to/ccmo_search.nsf/Searchform?OpenForm). GE-137 is a water-soluble 26-amino acid cyclic peptide labeled with a proprietary cyanine dye $\left(\lambda_{\max \text { ex }}=648 \mathrm{~nm}\right)$ with a high affinity $\left(K_{\mathrm{d}}=2 \mathrm{nM}\right)$ for human c-Met. We describe the stages of development of GE-137, as well as its safety, pharmacokinetics and imaging characteristics in healthy volunteers and patients at high risk of colorectal neoplasia, in conjunction with a customized colonoscopy imaging system. Because there is currently no commercially available colonoscope with the ability to detect both WL and near-infrared (NIR) fluorescent light, we developed a custom-built dual WL and fluorescent light (FL) endoscopic imaging system specifically for this project.

${ }^{1}$ Centre for Human Drug Research, Leiden, the Netherlands. ${ }^{2}$ GE Healthcare, Oslo, Norway. ${ }^{3}$ GE Global Research Centre, Niskayuna, New York, USA. ${ }^{4}$ Department of Gastroenterology and Hepatology, Leiden University Medical Center, Leiden, the Netherlands. ${ }^{5}$ Pathology Diagnostics Ltd., St. John's Innovation Centre, Cambridge, UK. ${ }^{6}$ Department of Pathology, Leiden University Medical Center, Leiden, the Netherlands. ${ }^{7}$ Department of Radiology, Leiden University Medical Center, Leiden, the Netherlands. ${ }^{8}$ GE Healthcare, Amersham, UK. Correspondence should be addressed to J.C.H.H. (j.c.h.hardwick@lumc.nl). 


\section{TECHNICAL REPORTS}

\section{RESULTS}

\section{Generation of a fluorescent c-Met binding probe}

The water-soluble 26-amino acid cyclic peptide component of the probe was selected from an M-13 phage-display library on the basis of its high affinity for the extracellular domain of human c-Met, its lack of competition with hepatocyte growth factor (HGF) ${ }^{14}$ and its lack of effect on HGF-stimulated proliferation in vitro (data not shown). We then conjugated this peptide (AGSCYCSGPPRFECWCYETEGT) with a linker peptide (GGGK) and subsequently to a modified Cy5 dye (Cy5 ${ }^{\star *}$ ) to yield GE-137 (AGSCYCSGPPRFECWCYETEGTGGGK-Cy5 ${ }^{\star *}$ (Fig. 1a)). This particular Cy5 derivative was selected from four cyanine dyes (Supplementary Fig. 1) on the basis of its improved brightness, resistance to photobleaching and low degree of binding to plasma proteins compared to the other dyes tested (data not shown). The fluorescence absorbance and emission spectra for GE-137 are shown in Figure 1b. The affinity of GE-137 for human c-Met was determined in vitro using a fluorescence polarization assay: $K_{\mathrm{d}}=3 \mathrm{nM}( \pm 0.5)$ (Fig. 1c).

Lyophilized (dry) GE-137 has a documented shelf life of 12 months when stored at $2-8{ }^{\circ} \mathrm{C}$. The chemical and physical stability of the reconstituted product (in solution) has been demonstrated for $24 \mathrm{~h}$ at $2-8{ }^{\circ} \mathrm{C}$ (Supplementary Table 1 ). Furthermore, the compound
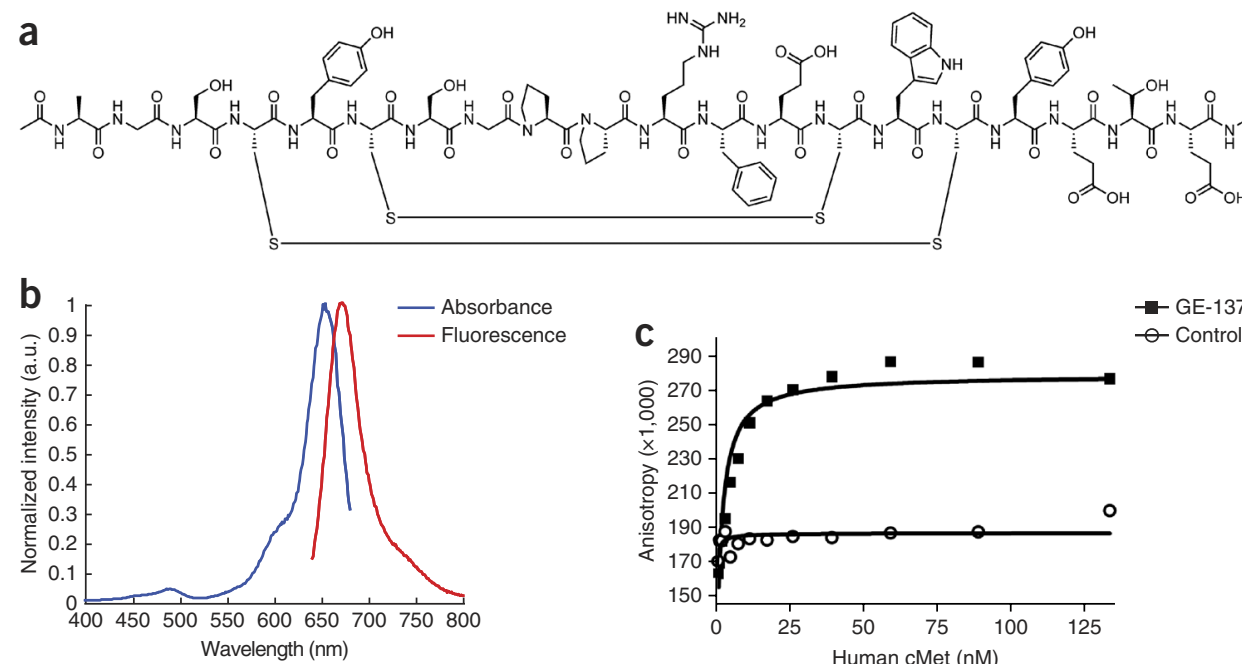<smiles>CC(=O)NCCCCC(NC(=O)CNC(=O)CNC(=O)CNC(=O)C(O)NC(C)=O)C(N)=O</smiles>
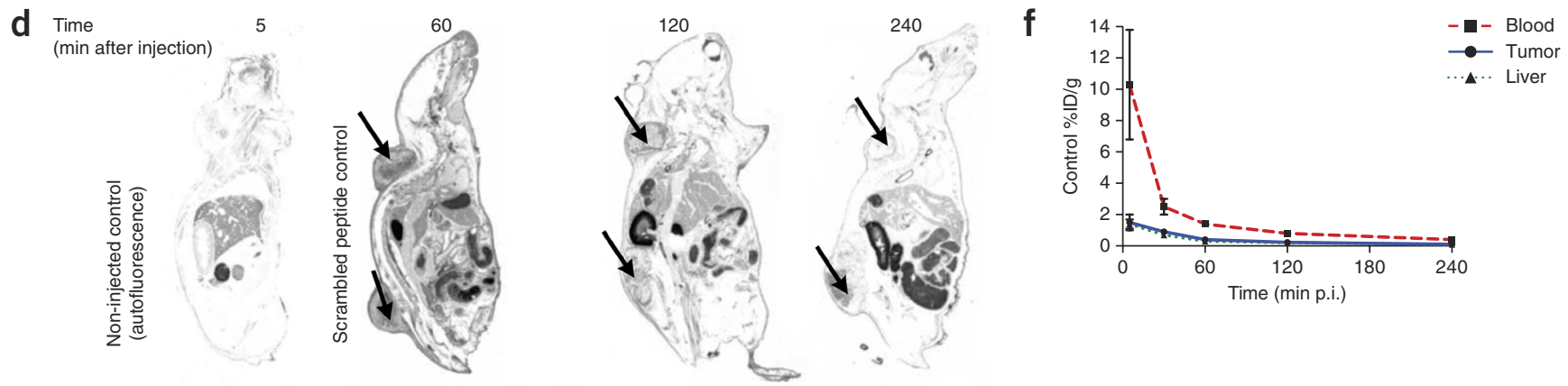

e

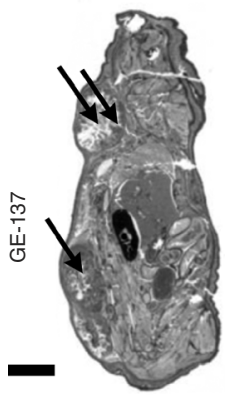

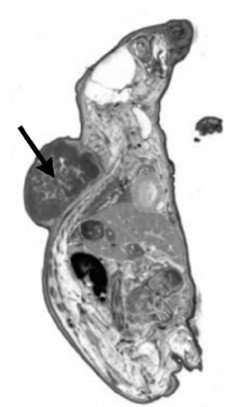

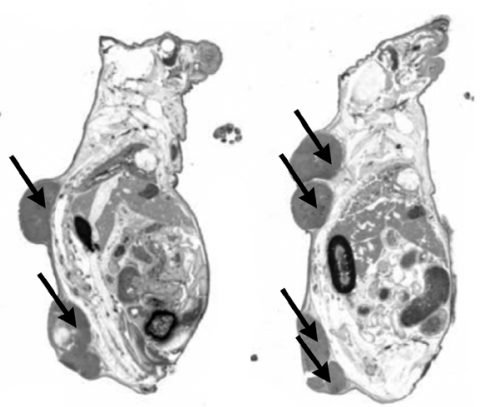

g

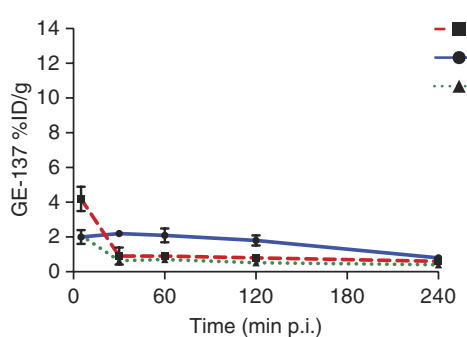

Figure 1 GE-137 chemical and optical properties and testing in a rodent model of colon cancer. (a) Chemical structure of the probe. (b) Fluorescence absorbance and emission spectra of the probe. (c) Fluorescence polarization analysis demonstrating binding of the contrast agent (black squares) to cMet with a $K_{d}$ of $\sim 3 \mathrm{nM}( \pm 0.5)$. The scrambled peptide control (white circles) showed no binding in the concentration range tested (0-150 $\mathrm{nM}$ ). $(\mathrm{d}, \mathrm{e})$ Representative fluorescence scanned cryosections of whole tumor bearing nude mice at various time points after injection of Cy $5^{* *}$-labeled scrambled peptide control (d) or GE-137 (e). The arrows indicate the tumor locations. A non-injected control was also included (d, leftmost cryosection). The time points after injection were, from left to right, 5, 60, 120 and $240 \mathrm{~min}$, respectively. Scale bar, $1 \mathrm{~cm}$. Images are displayed on a linear 'negative' gray scale in which black indicates high fluorescence signal. $(\mathbf{f}, \mathbf{g})$ Graphs to show the results of quantification of the fluorescence (expressed as a percentage of the injected dose per gram of tissue (\%/D/g)) in blood and homogenates of liver and tumor tissue at 5, 30, 60, 120 and 240 min after injection of Cy ${ }^{* *}$-labeled scrambled peptide control (f) or GE-137 (g). a.u., arbitrary units. Error bars show means \pm s.e.m. 
Figure 2 Experimental system for combined WL and NIR fluorescence colonoscopy in humans. (a) Fluorescence image showing the method of estimation of colonic wall background fluorescence. A fluorescent probe standard inserted via the working channel of the endoscope (indicated with an arrow) is shown approximated to the colonic wall (see methods). (b) Screenshot of the simultaneous WL and FL endoscopic images as seen on the monitor during endoscopy. (c) The customized fluorescence colonoscopy system. The standard fiber-colonoscope is shown attached via the eyepiece to the large imaging head supported by an adjustable arm.

is photostable under ambient light conditions for several hours and is not substantially bleached by intense light exposure even after several minutes (the fluorescence half-life is $7 \mathrm{~min}$ in tissue samples illuminated under worst-scenario conditions $\left(300 \mathrm{~mW} \mathrm{~cm}^{-2}\right)$ ) (Supplementary Fig. 2).

\section{Preclinical efficacy studies}

The quantitative biodistribution, pharmacokinetics, binding specificity and qualitative fluorescence of GE-137 were assessed in a xenograft mouse model of CRC using subcutaneous injection of the c-Met-expressing human CRC cell line HT29. Cryosections of whole mice were made and fluorescence scans performed using a method we have described previously ${ }^{15}$. Intravenously administered GE-137 accumulates in the c-Met-expressing tumor xenografts and washes out of other tissues to leave a fluorescent signal in the tumors and kidneys 120 and $240 \mathrm{~min}$ after injection, whereas the Cy5 $5^{\star *}$-labeled scrambled peptide control is only visible in the kidneys (Fig. 1d,e). Fluorescence pharmokinetics and qualitative fluorescence imaging were performed in vivo (Supplementary Fig. 3a,b). Co-administration of GE-137 together with an excess of unlabeled peptide led to a reduction in tumor uptake of the agent (Supplementary Fig. 3c,d). In the same mouse model, the quantitative biodistribution of the probe was measured in blood and homogenates of the liver, kidney, muscle and c-Met-expressing tumor xenografts at 5, 30, 60, 120 and 240 min after intravenous administration of GE-137 or the control (Fig. 1f,g). GE-137 accumulates in the c-Met-expressing tumor xenografts, whereas the labeled scrambled peptide control does not. Further in-vivo fluorescence imaging was performed in a second mouse model designed to more closely replicate the clinical situation. In the first mouse model, large spherical subcutaneously xenografted tumors were imaged against a background of muscle that doesn't express c-Met. In the clinical situation, small flat polyps must be detected against a background of normal colon, which expresses c-Met at low levels and may have fecal remnants with native fluorescence. Therefore, in the second mouse model, subcutaneous HT-29 and HCT-15 tumor xenografts from mice injected with GE-137 or a labeled scrambled peptide control were surgically removed and thin slices of the tumor were imaged against a background of surgically opened normal mouse colon. The tumor-to-colon ratio of fluorescence was measured and images were assessed by blinded observers (Supplementary Fig. 4). These preclinical studies suggest that GE-137 accumulates specifically in human c-Met-expressing colorectal tumors and that GE-137 at a dose of $0.18 \mathrm{mg} \mathrm{kg}^{-1}$ will provide sufficient contrast to enable c-Met-rich lesion detection against a background of normal colon in humans.

\section{Safety studies}

The probe was extensively tested for safety with in vitro genotoxicity and phototoxicity studies. Biodistribution, pharmacology and general toxicity studies were performed in rats and cynomolgus monkeys in a

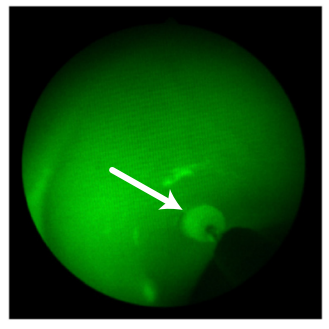

b

C

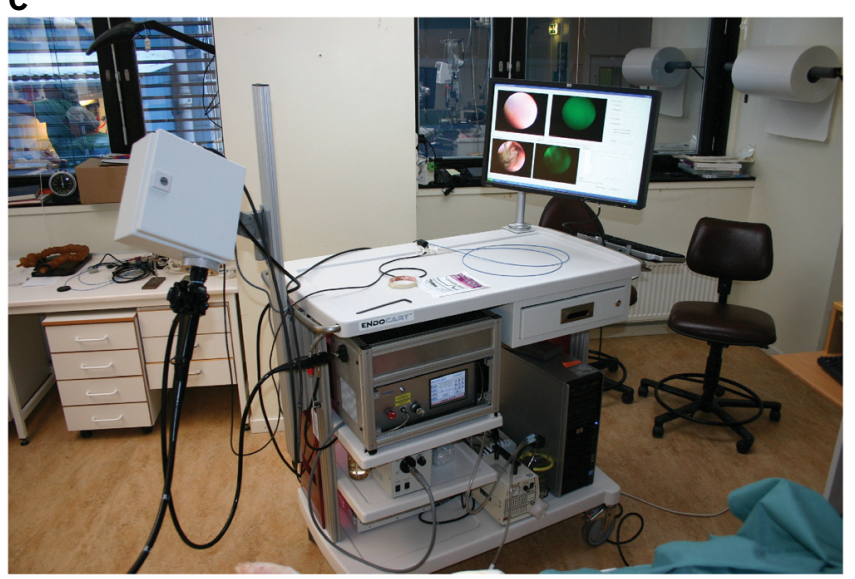

single-dose and repeat-dose studies. Specific toxicity was investigated with genotoxicity tests, spermatogenesis evaluation and cardiovascular toxicity testing in cynomolgus monkeys. No adverse effects were seen in any of these studies (data not shown). When the no observed adverse effect level (NOAEL) was expressed as human equivalent dose on the basis of body surface area, this corresponded to $15.5 \mathrm{mg} \mathrm{kg}^{-1}$. This suggested that a single intravenous (i.v.) dose of up to $0.36 \mathrm{mg} \mathrm{kg}^{-1}$, (twice the expected efficacious clinical imaging dose) would be well tolerated in humans. A single i.v. administration of GE-137 (0.02-0.18 $\left.\mathrm{mg} \mathrm{kg}^{-1}\right)$ was well tolerated and seemed to be safe in both healthy volunteers and subjects at high risk of colorectal neoplasia (total $n=31$ ) (Supplementary Table 2).

The optimal dose and optimal time interval between GE-137 administration and endoscopy, or optimal imaging time point, was estimated by performing colon wall fluorescence measurements (Fig. 2a) in healthy volunteers at repeated sigmoidoscopy using a prototype fluorescence colonoscope (Fig. 2b,c) after GE-137 administration. The background clearance half-life was calculated from these measurements and was $\sim 2 \mathrm{~h} 30 \mathrm{~min}$ at all doses (data not shown). The optimal imaging time point $(3 \mathrm{~h})$ was determined by adding the time to peak-signal ( $30 \mathrm{~min}$ (data not shown)) to one background clearance half-life. The intensity and uniformity of the texture of colon wall fluorescence observed were higher than expected from the preclinical model (data not shown). It was therefore estimated that a dose reduction to $70 \%$ of the optimal imaging dose calculated from the preclinical models (from $0.18 \mathrm{mg} \mathrm{kg}^{-1}$ to $0.13 \mathrm{mg} \mathrm{kg}^{-1}$ ) would be optimal for imaging subjects with a high suspicion of CRC.

GE-137 is renally cleared, and the exposure derived from area under the curve (AUC) data was dose-linear (Supplementary Fig. 5). The clearance rate was approximately $0.131 \mathrm{~kg}^{-1} \mathrm{hr}^{-1}$ and comparable between dose groups. At a dose of $0.13 \mathrm{mg} \mathrm{kg}^{-1}$, the observed plasma concentrations were $0.12-0.16 \mathrm{mg} \mathrm{l}^{-1}$ at $3 \mathrm{~h}$ after administration. 
Figure 3 Simultaneous WL and FL images of representative lesions of the various morphological and histological subtypes found. (a-c) The lesions shown are clearly visible in WL and show clearly increased fluorescence. (d) A lesion that, although it is visible in WL, has enhanced visibility in FL. (e,f) Images representative of the nine lesions that were only visible in FL. Polyps are indicated by the white arrows. (a) 2-cm pedunculated (Paris 0-Ip) tubular adenoma. (b) 4-cm subpedunculated (Paris 0-Ips) tubulovillous adenoma. (c) 2-cm sessile (Paris 0-Is) serrated polyp. (d) $5-\mathrm{mm}$ flat elevated (Paris 0-Ila) tubular adenoma. $(\mathrm{e}, \mathrm{f})$ flat (Paris $0-\mathrm{IIb})$ tubular adenomas, 5 and $4 \mathrm{~mm}$ in diameter, respectively. (g) Graph showing the relationship between the degree of fluorescence and the histological diagnosis. (h) Graph showing the relationship between c-Met expression and the degree of

fluorescence. Error bars are means \pm s.e.m. n.s., not significant; ${ }^{* *} P<0.001$; mixedmodel analysis of variance. (i) Graph showing the relationship between c-Met expression and histological diagnosis. Error bars are means \pm s.e.m. n.s., not significant; $* * * P<0.001$, mixed-model analysis of variance.

\section{Pilot study in patients}

In 15 patients at high risk of colorectal neoplasia a total of 101 lesions were detected; were detected during first-pass inspection with WL, and an additional 22 were detected during second-pass inspection with dual WL/FL. Of these, 17 were only visible using FL imaging (Fig. 3 and Supplementary Table 3). The additional lesions detected with FL tended to be small $(<6 \mathrm{~mm})$ and have non-polypoid morphology (Fig. 3e,f).

Fluorescence relative to background was assessed during the live imaging for all the 101 lesions described above as well as the areas selected for additional biopsies from normal mucosa. Fluorescence was clearer in a dynamic real-time setting (Supplementary Videos 1-4) than it is in a still image. Most normal mucosa showed background fluorescence, whereas most adenomas had clearly increased fluorescence and none displayed background levels of fluorescence (Fig. 3g) The majority of hyperplastic polyps had increased fluorescence. The proportion of lesions from each histological category with increased fluorescence over background was adenoma 100\% (47/47), hyperplastic polyp 78\% (33/42) and normal histology 19\% (8/41). Overall, fluorescence findings in vivo were highly significantly associated with the histology (Fig. 3g, $P=0.00003$, Fisher's exact test). Fluorescence

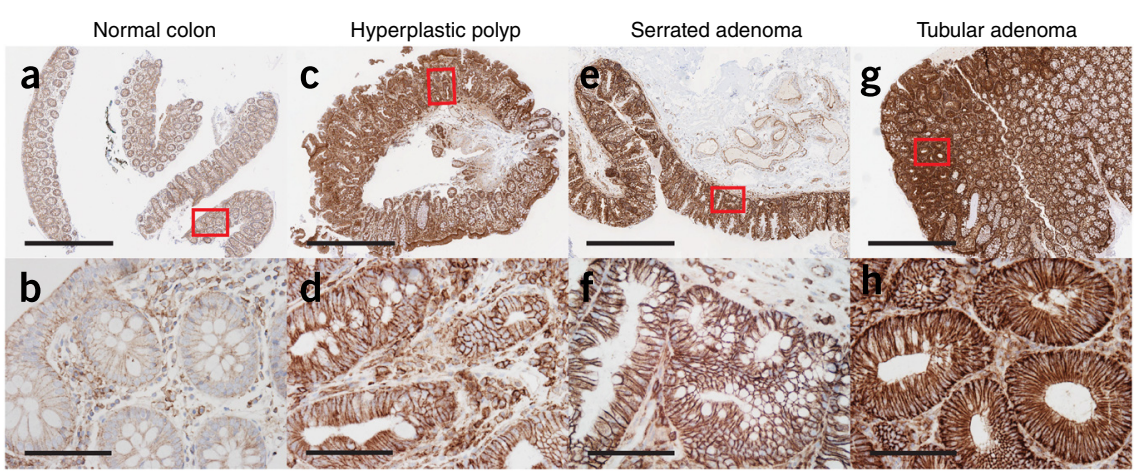

is also significantly associated with c-Met expression, as estimated by immunohistochemical analysis $(P=0.0025$, mixed model analysis of variance), suggesting that the fluorescent probe indeed binds c-Met in vivo in humans (Fig. 3h).

Histological analysis was possible for 100 of the 101 lesions removed; 47 were adenomas, 42 hyperplastic polyps and 11 were classified as normal. Twelve (26\%) of the adenomas were found on the second-pass dual WL/FL examination, of which three were visible in WL after closer inspection and nine (19\%) were only visible in FL. Twelve lesions (nine hyperplastic polyps, three normal) detected with WL imaging did not show increased fluorescence; of these only three (two hyperplastic polyps, one normal) were not visible in FL at all, the rest being visible in the fluorescence images owing to their contours. The histological classification of lesions was significantly associated with the level of fluorescence seen in them in vivo (Fig. $\mathbf{3 g}$, $P=0.00003$, Fisher's exact test). Both imaging modalities were equally likely to identify false positive lesions (eight in each modality).

All available biopsies from detected lesions $(n=100)$, as well as two biopsies taken from endoscopically normal mucosa from each patient, were analyzed for c-Met expression by immunohistochemical

Figure 4 c-Met expression analysis by immunohistochemistry using an antibody specific for the extracellular domain of c-Met. (a,b) Normal colonic mucosa. (c,d) Hyperplastic polyp. (e,f) Serrated adenoma. (g,h) Tubular adenoma. Scale bars (a,c,e,g), $1 \mathrm{~mm}$; (b,d,f,h), $100 \mu \mathrm{m}$. The lower images show the area within the red rectangle on the upper images at higher magnification. Controls omitting the primary antibody showed no staining (data not shown). 

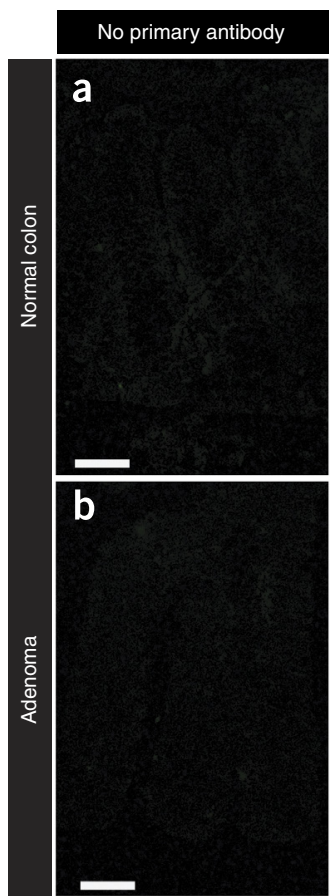
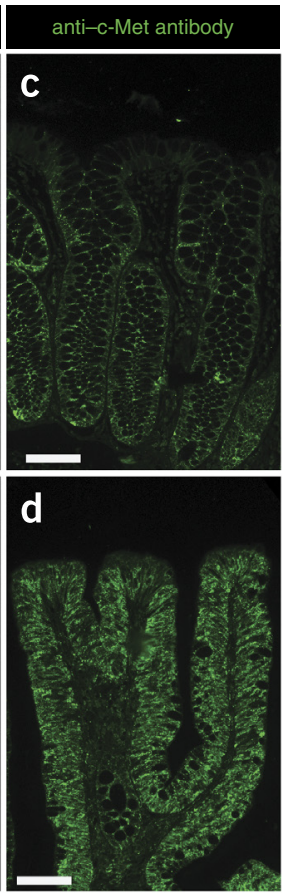
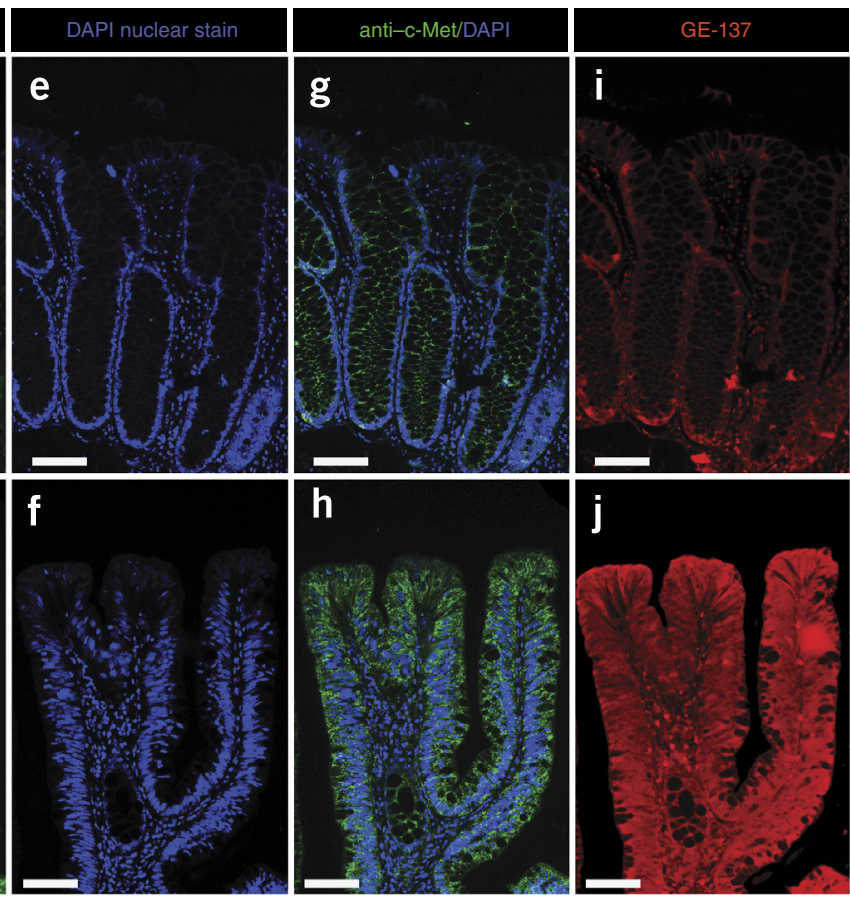
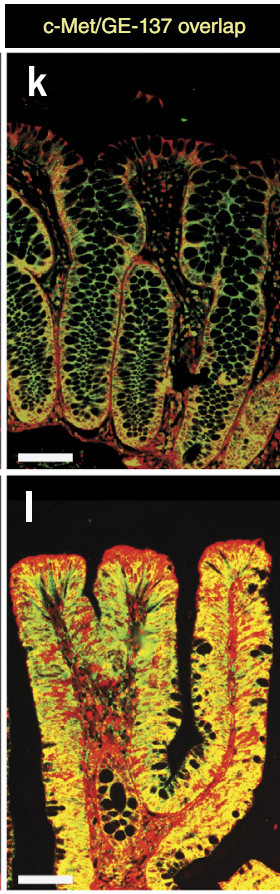

Figure 5 c-Met expression in normal colon and an adenomatous polyp as detected by immunohistochemistry and GE-137. (a,b) Controls omitting the primary antibody showed no staining. (c,d) Fluorescence immunohistochemistry for c-Met labeled with fluorescein isothiocyanate (FITC), shown in green. (e-h) Nuclei stained with DAPI, shown in blue (e,f), and DAPI combined with immunohistochemistry for c-Met (g,h). (i,j) Direct fluorescence microscopy to visualize GE-137, shown in red. (k,I) The overlap between immunohistochemical staining for c-Met and the presence of GE-137, shown in yellow. All images are taken from the same tissue sections from biopsies taken after GE-137 administration. (a,e,g,i,k) Normal colonic mucosa. (b,d,f,h,I) Tubulovillous adenoma. Scale bars, $100 \mu \mathrm{m}$.

analysis using an antibody specific for the extracellular domain of c-Met. Whereas c-Met is expressed in normal colon tissue and is evenly expressed along the crypt axis on the epithelial cell membrane, it is significantly more highly expressed in both hyperplastic and adenomatous polyps (Fig. 3i, $P<0.001$ for both, mixed-model analysis of variance; and Fig. 4).

Biopsies from detected lesions and normal mucosa from the patient study were also analyzed ex vivo for direct fluorescence from the probe while embedded in paraffin. There is more fluorescence detectable in lesions with abnormal histology (adenomatous or hyperplastic) than in normal tissue with a target-to-background ratio of $2.3 \pm 1.1$ (mean \pm s.d.). Fluorescence microscopy was also performed on tissue sections from normal colon and lesions from the patient study. Fluorescence immunohistochemistry for c-Met in representative images from normal colon and from an adenomatous polyp can be compared to direct fluorescence from GE-137 in the same sections and in the overlay of c-Met immunohistochemical analysis and direct fluorescence measurements from GE-137 (Fig. 5). There is clearly increased signal from GE-137 in adenomatous tissue, and this overlaps with the c-Met expression from the immunohistochemical analyses. The direct fluorescence from the probe is less clearly on the cell membrane than the c-Met expression from immunohistochemistry. We have previously documented that the process of fixation influences the distribution of fluorescent probes for membrane-bound receptors ${ }^{16}$, so this is likely to be a tissue-fixation artifact.

\section{DISCUSSION}

A variety of new endoscopic imaging techniques aimed at enhancing the ability to detect and characterize colorectal lesions have been described ${ }^{17}$. They can be broadly characterized into either wide-field 'red-flag' techniques or high-resolution 'optical biopsy' techniques. The latter (confocal laser endomicroscopy, endocytoscopy, and optical coherence tomography) are not suitable for lesion detection, as they only microscopically examine a few square millimeters of the colon at a time.

Wide-field techniques using light alone, such as narrow-band imaging (NBI), digital I-scan, Fujinon Intelligent Color Enhancement (FICE), and autofluorescence imaging (AFI), are widely used, but there are as yet no large randomized trials showing an advantage of these modalities over high-definition WL endoscopy for lesion detection ${ }^{18,19}$.

Topical application of nonspecific dyes has been used both in conjunction with WL (Methylene blue, indigo carmine) and fluorescence endoscopy (hexaminolevulinate) ${ }^{20}$. An inherent limitation of topical application is that complete mucosal coverage is rarely achievable, and mucus consistency and bowel cleanliness detrimentally affect mucosal binding.

Topical application of fluorescently labeled antibodies has been investigated using fluorescein-labeled monoclonal antibodies against carcinoembryonic antigen (CEA) and a modified fiber colonoscope in humans ${ }^{21}$, and using i.v. administration of fluorescein-labeled antibodies against epidermal growth factor receptor (EGFR) or Alexa Fluor 488-labeled antibodies against vascular endothelial growth factor (VEGF) in mice in conjunction with confocal laser endomicroscopy ${ }^{22,23}$. Problems associated with the use of fluorescently tagged antibodies include a long clearance half-life after injection and concerns regarding immunogenicity ${ }^{24}$. In this respect, low-molecularweight small peptides have a number of potential advantages, such as favorable pharmacokinetic and tissue distribution patterns, increased permeability, lower toxicity, lower immunogenicity, and flexibility in chemical modification ${ }^{25}$. 


\section{TECHNICAL REPORTS}

Topical application of a fluorescein-labeled heptapeptide probe isolated from a phage library has been investigated in conjunction with endoscopic confocal microscopy in humans ${ }^{11}$. The exact target for this heptapeptide has yet to be determined, so any potential downstream signaling due to peptide binding has not been evaluated. This study made use of endoscopic confocal microscopy, in which only a few square millimeters of colon can be examined, and fluorescein, a suboptimal fluorophore for use in the colon ${ }^{26}$.

Further studies of topical application of fluorescent imaging probes in the colon in mice include a Cy5.5-conjugated octapeptide probe combined with a near-infrared wide-field fiber optic catheter ${ }^{26}$ and an enzymatically activated green fluorescent probe (gGlu-HMRG) and a modified bronchoscope ${ }^{27}$.

Probe-based studies in the human colon have generally used either fluorescein or Alexa Fluor 488 as the fluorophore. These green fluorescent fluorophores fluoresce maximally at around $520 \mathrm{~nm}$. Autofluorescence of colonic mucosa also occurs at similar wavelengths ${ }^{28}$. Many authors recommend the use of fluorophores in the NIR spectrum, in which colon autofluorescence is negligible ${ }^{26}$.

In summary, previously investigated techniques have all suffered from one or more of potential limitations, including the use of: nonspecific dyes; topically applied dyes or probes for which complete mucosal coverage is rarely achievable; antibody probes with potential for immunogenicity and unfavorable pharmacokinetics; peptide probes with an unknown target and thus potential effects on downstream signaling; fluorophores whose fluorescence spectrum overlaps with colon autofluorescence; or endoscopy techniques unsuitable for wide-area surveillance.

GE-137 is a small (26 amino acids) fluorescently labeled peptide agent with high affinity for a known molecular target, human c-Met, and with no effect on downstream signaling, conjugated with a near-infrared fluorophore. Peptides for the peptide component were selected for their ability to bind c-Met without having effects on downstream signaling. Fluorophores for the fluorophore component were selected to have spectral characteristics that do not overlap with colon autofluorescence, as well as to provide minimal photobleaching and maximum brightness. GE-137 was selected from a panel of peptide and fluorophore combinations for its optimal combination of high affinity for c-Met, minimal binding to plasma proteins and a favorable safety profile and pharmacodynamics in animal models. These initial studies in humans suggest that GE-137 is safe and may enhance visualization of colonic polyps.

One of the frequently stated goals of advanced imaging techniques in the colon is the ability to characterize lesions in vivo to avoid the added costs and risks associated with the removal of benign hyperplastic polyps ${ }^{29}$. However, the appreciation of the malignant potential of serrated polyps makes it important that molecular imaging techniques also detect these lesions ${ }^{30}$. In this study, we identified 36 hyperplastic lesions and eight serrated polyps. Most of these were visible in fluorescent mode (94\% and $100 \%$ respectively) and the majority (78\% and $87 \%$ respectively) showed increased fluorescence (Supplementary Table 3).

Although several studies confirm c-Met overexpression in CRCs, the number of adenomas studied has been small and hyperplastic polyps have not been included ${ }^{13,31}$. We find that c-Met expression is significantly increased in both hyperplastic and adenomatous tissue. This confirms that c-Met is a suitable target for the molecular detection of colorectal neoplasia.

This study has a number of limitations. The low number of patients included in this pilot study precluded randomization between WL and fluorescence endoscopy as the first-pass endoscopic technique. It is therefore not possible to ascertain whether fluorescence endoscopy leads to the detection of substantial numbers of additional polyps. Second, as there are currently no commercial fluorescence video colonoscopes that can visualize Cy5, a customized fluorescence endoscopy system had to be developed especially for this study using a fiber colonoscope. Future studies using high-definition fluorescent video colonoscopy, once this is available, are needed to confirm the encouraging but preliminary findings presented here.

In conclusion, intravenous administration of GE-137 is feasible and safe in humans. It accumulates specifically in c-Met-rich premalignant lesions and can potentially enhance polyp detection at colonoscopy.

\section{METHODS}

Methods and any associated references are available in the online version of the paper.

Note: Any Supplementary Information and Source Data files are available in the online version of the paper.

\section{ACKNOWLEDGMENTS}

The authors would like to acknowledge the contributions of T. Attramadal, E. Johannesen and H. Dirven, who were the Project Leaders at GE Healthcare in the screening, lead selection and the translational/early clinical phases of the project, respectively. GE Healthcare AS received support for the GE-137 project from The Research Council of Norway grant no. 192984/BIA and SkatteFUNN grant no. 201108. F.W.B.v.L. and T.B. were supported by the The Netherlands Organisation for Scientific Research (NWO) VIDI (grant no. STW BGT11272) and a Postdoctoral Molecular Imaging Scholarship from the Education and Research Foundation for Nuclear Medicine and Molecular Imaging.

\section{AUTHOR CONTRIBUTIONS}

J.B., I.M.C.K. and P.B.G. designed the pharmacological studies; B.I., M.S.-N. and L.-I.Ø. developed the probe and the drug product; and R.B., R.M.B., S.A.M., A.H. and G.T.D. conducted the preclinical testing. L.S. and A.J.K. performed recruitment and safety testing for the clinical studies. M.L.d.K. performed the statistical analyses. H.M. performed the histopathological analysis of polyps. M.V.W. performed the c-Met immunohistochemical analysis. F.W.B.v.L., P.W.V. and T.B. performed the ex vivo direct fluorescence imaging studies. A.H., S.Y. and G.T. designed the method for assessing the imaging characteristics of the probe and developed the colonoscopy system. P.B.G., A.M.J.L. and J.C.H.H. designed the clinical studies, A.M.J.L. and J.C.H.H. performed the clinical studies, and J.C.H.H. wrote the manuscript.

\section{COMPETING FINANCIAL INTERESTS}

The authors declare competing financial interests: details are available in the online version of the paper.

Reprints and permissions information is available online at http://www.nature.com/ reprints/index.html.

1. Jemal, A. et al. Global cancer statistics. CA Cancer J. Clin. 61, 69-90 (2011).

2. Atkin, W.S. et al. Once-only flexible sigmoidoscopy screening in prevention of colorectal cancer: a multicentre randomised controlled trial. Lancet 375 , 1624-1633 (2010).

3. Baxter, N.N. et al. Association of colonoscopy and death from colorectal cancer Ann. Intern. Med. 150, 1-8 (2009).

4. Brenner, $\mathrm{H}$. et al. Protection from right- and left-sided colorectal neoplasms after colonoscopy: population-based study. J. Natl. Cancer Inst. 102, 89-95 (2010).

5. Kaminski, M.F. et al. Quality indicators for colonoscopy and the risk of interval cancer. N. Engl. J. Med. 362, 1795-1803 (2010).

6. Kahi, C.J., Hewett, D.G., Norton, D.L., Eckert, G.J. \& Rex, D.K. Prevalence and variable detection of proximal colon serrated polyps during screening colonoscopy. Clin. Gastroenterol. Hepatol. 9, 42-46 (2011).

7. van Rijn, J.C. et al. Polyp miss rate determined by tandem colonoscopy: a systematic review. Am. J. Gastroenterol. 101, 343-350 (2006).

8. Clapper, M.L. et al. Detection of colorectal adenomas using a bioactivatable probe specific for matrix metalloproteinase activity. Neoplasia 13, 685-691 (2011).

9. Marten, K. et al. Detection of dysplastic intestinal adenomas using enzyme-sensing molecular beacons in mice. Gastroenterology 122, 406-414 (2002). 
10. Endlicher, $\mathrm{E}$. et al. Hexaminolevulinate-induced fluorescence endoscopy in patients with rectal adenoma and cancer: a pilot study. Gastrointest. Endosc. 60, 449-454 (2004).

11. Hsiung, P.-L. et al. Detection of colonic dysplasia in vivo using a targeted heptapeptide and confocal microendoscopy. Nat. Med. 14, 454-458 (2008).

12. Fearon, E.R. \& Vogelstein, B. A genetic model for colorectal tumorigenesis. Cell 61, 759-767 (1990).

13. Di Renzo, M.F. et al. Overexpression and amplification of the met/HGF receptor gene during the progression of colorectal cancer. Clin. Cancer Res. 1, 147-154 (1995).

14. Nanjappan, A.K.S. et al. Peptides that specifically bind HGF receptor (cMet) and uses thereof. Patent no. WO 2004/078778 A2. (2004).

15. Moestue, S. et al. Whole-body section fluorescence imaging-a novel method for tissue distribution studies of fluorescent substances. Contrast Media Mol. Imaging 4, 73-80 (2009).

16. van den Berg, N.S., Buckle, T., Kuil, J., Wesseling, J. \& van Leeuwen, F.W.B. Immunohistochemical detection of the CXCR4 expression in tumor tissue using the fluorescent peptide antagonist Ac-TZ14011-FITC. Transl. Oncol. 4, 234-240 (2011).

17. Kiesslich, R., Goetz, M., Hoffman, A. \& Galle, P.R. New imaging techniques and opportunities in endoscopy. Nat. Rev. Gastroenterol. Hepatol. 8, 547-553 (2011).

18. Adler, A. et al. A prospective randomised study on narrow-band imaging versus conventional colonoscopy for adenoma detection: does narrow-band imaging induce a learning effect? Gut 57, 59-64 (2008).

19. Kuiper, T. et al. Endoscopic trimodal imaging detects colonic neoplasia as well as standard video endoscopy. Gastroenterology 140, 1887-1894 (2011).

20. Mayinger, B., Neumann, F., Kastner, C., Haider, T. \& Schwab, D. Hexaminolevulinateinduced fluorescence colonoscopy versus white light endoscopy for diagnosis of neoplastic lesions in the colon. Endoscopy 42, 28-33 (2010).
21. Keller, R., Winde, G., Terpe, H.J., Foerster, E.C. \& Domschke, W. Fluorescence endoscopy using a fluorescein-labeled monoclonal antibody against carcinoembryonic antigen in patients with colorectal carcinoma and adenoma. Endoscopy 34 801-807 (2002).

22. Foersch, S. et al. Molecular imaging of VEGF in gastrointestinal cancer in vivo using confocal laser endomicroscopy. Gut 59, 1046-1055 (2010).

23. Goetz, M. \& Wang, T.D. Molecular imaging in gastrointestinal endoscopy. Gastroenterology 138, 828-833 (2010).

24. Wu, A.M. \& Olafsen, T. Antibodies for molecular imaging of cancer. Cancer J. 14 191-197 (2008)

25. Chen, K. \& Chen, X. Design and development of molecular imaging probes Curr. Top. Med. Chem. 10, 1227-1236 (2010).

26. Liu, Z., Miller, S.J., Joshi, B.P. \& Wang, T.D. In vivo targeting of colonic dysplasia on fluorescence endoscopy with near-infrared octapeptide. Gut 62, 395-403 (2013).

27. Mitsunaga, M. et al. Fluorescence endoscopic detection of murine colitis-associated colon cancer by topically applied enzymatically rapid-activatable probe. Gut $\mathbf{6 2}$, 1179-1186 (2013).

28. Imaizumi, K. et al. Dual-wavelength excitation of mucosal autofluorescence for precise detection of diminutive colonic adenomas. Gastrointest. Endosc. 75, 110-117 (2012).

29. Rex, D.K. Reducing costs of colon polyp management. Lancet Oncol. 10 1135-1136 (2009)

30. Leggett, B. \& Whitehall, V. Role of the serrated pathway in colorectal cancer pathogenesis. Gastroenterology 138, 2088-2100 (2010).

31. Wielenga, V.J. et al. Expression of c-Met and heparan-sulfate proteoglycan forms of CD44 in colorectal cancer. Am. J. Pathol. 157, 1563-1573 (2000). 


\section{ONLINE METHODS}

GE-137 safety. In vitro safety testing. GE-137 was tested in vitro in the Ames and MLA (mouse lymphoma assay) assays for evaluation of genotoxic hazard, the 3T3 NRU (neutral red uptake) assay for assessment of phototoxic hazard, the hERG assay for assessment of potential of drug-induced QT interval prolongation, and in a proliferation assay for evaluation of interference with c-Met signaling.

Animal safety testing. GE- 137 has no or very low affinity for mouse c-Met and approximately 100-fold lower affinity for dog c-Met compared to human c-Met. The reported amino acid sequence homology for c-Met from mouse, rat, dog and Macaca fascicularis (cynomolgus monkey) is approximately $89.5 \%, 88.1 \%, 91.0 \%$ and $98.2 \%$, respectively, when compared to human c-Met. The cynomolgus monkey was selected as the relevant non-rodent species for safety studies.

General toxicity of GE-137 Injection was evaluated in an expanded acute single-dose toxicity study in male and female rats. The nonclinical safety studies in cynomolgus monkey were conducted in compliance with the Animal Welfare Act, the Guide for the Care and Use of Laboratory Animals, and the Office of Laboratory Animal Welfare (USA) under a protocol reviewed and approved by the Preclinical Study Review Team, Global Biology, GE Healthcare. The nonclinical safety study in the rat were conducted in compliance with the Animal Welfare Act, the Guide for the Care and Use of Laboratory Animals, and the Office of Laboratory Animal Welfare (USA) under a protocol reviewed and approved by the Preclinical Study Review Team, Global Biology, GE Healthcare. Animals received injections of up to 50 times the expected maximum clinical dose $(0.36 \mathrm{mg} / \mathrm{kg}$ body weight $)$ scaled for body surface area and were observed up to 4 weeks afterwards. In a 14-day repeat-dose toxicity study in sexually mature cynomolgus monkeys ( 4 to 9 years of age for males and 3-7 years of age for females). The high dose-group in this study received daily doses of $16.8 \mathrm{mg} \mathrm{GE}-137$ per kg of body weight. As part of the study, the genotoxic risk of GE-137 injection was assessed in the micronucleus test of peripheral blood. In addition, a spermatogenic staging evaluation of the testes and epididymis was carried out to assess the potential adverse effects on the male reproductive system. Animals were monitored up to 4 weeks after the last administered dose.

The potential of GE-137 injection to cause effects on the cardiovascular system was assessed in the conscious male cynomolgus monkey in doses up to $48 \mathrm{mg} / \mathrm{kg}$ of body weight. Changes in ECG, blood pressure and heart rate were assessed. When the NOAEL was expressed as human equivalent dose based on body surface area, this corresponds to $15.5 \mathrm{mg} / \mathrm{kg}$ body weight of GE-137. The ${ }^{14} \mathrm{C}$-biodistribution studies in rats and cynomolgus monkeys indicated that GE-137 does not cross the blood-brain barrier. The effect of GE137 injection on the central nervous system was tested in the modified Irwin screen in the male rat with injection of up to $48 \mathrm{mg} \mathrm{GE}-137$ per mg/kg of body weight. The effects of GE-137 injection on respiratory function as indicated by tidal volume, respiration rate, and minute volume were also assessed in the male rat in a head-out plethysmography study with doses up to $48 \mathrm{mg}$ GE-137 per mg/kg of body weight.

Studies in mouse xenografts. Animal models. The use of the mice was approved by the Norwegian Animal Research Authority (NARA) (Forsøksdyrutvalget). No formal statistical analysis was performed to estimate sample size. The animals were not formally randomized to a particular intervention. Female $\mathrm{BALB} / \mathrm{c} / \mathrm{A}$ nude (Bom) mice were housed in individually ventilated cages (IVC, Scanbur BK) with HEPA filtered air with ad libitum access to 'Rat and Mouse nr. 3 Breeding' diet (Scanbur BK).

Two colon cancer cell lines were used, HCT-15 and HT29, both derived from human colon carcinomas and acquired directly from the ATCC (Manassas, VA). HCT-15 cells: ATCC (CCL-225). HT-29 cells: ATCC (HTB-38). The cell lines were authenticated by STR profiling and tested for mycoplasma contamination by the ATTC before delivery.

The two cancer cell lines have different expression levels of the target cMet. One relatively high (HT29, termed 'high expression tumor') and the other relatively low (HCT-15, termed 'low expression tumor'). cMet expression levels were determined via by ELISA in both tumor types. The cell lines are tumorigenic when inoculated subcutaneously into nude mice. Cells were grown and prepared for subcutaneous inoculation in RPMI (Sigma, cat. no. R0883) with $10 \%$ serum and penicillin/streptomycin.

Stocks were made at passage number four (P4) and frozen down for storage in liquid nitrogen at $2 \times 10^{7}$ cells per vial in the culture media containing $5 \%$ DMSO. On the day of the transplantation, the cells were thawed quickly in a $37^{\circ} \mathrm{C}$ water bath $(\sim 2 \mathrm{~min})$, washed and resuspended in PBS (centrifugation at 2,000 r.p.m. for $10 \mathrm{~min}$ ). Thorough mixing of cells in the vials was ensured every time cells were aspirated into the dosing syringe. Volumes of $0.1 \mathrm{ml}$ of cell suspension were injected subcutaneously (s.c.) over the shoulder and back using a fine-bore needle $(25 \mathrm{G})$ while the animals were under light gas anesthesia. The animals were then returned to their cages and the tumors were allowed to grow for 13-17 d. The animals were allowed an acclimatization period of at least $5 \mathrm{~d}$ before the inoculation procedure.

Quantitative biodistribution of contrast agent and negative control in tumor-bearing BALB c/A nude mice. Whole blood sampled from the vena cava, $\sim 0.7 \mathrm{ml}$ per animal, was centrifuged for preparation of samples for plasma analysis, whereas organs were collected, snap frozen and stored at $-80^{\circ} \mathrm{C}$ until the day of analysis. Liver and kidneys were homogenized in PBS $\left(0.4 \mathrm{~g} \mathrm{ml}^{-1}\right)$ using a Polytron homogenizer. Tumor and muscle were crushed in liquid nitrogen, buffer with detergent was added (RIPA buffer, Santa Cruz), followed by homogenization using a Dounce glass homogenizer. For preparation of standard curve samples, blood plasma and homogenized tissue were spiked with known amounts of substance in a relevant concentration range. Standard curves were then generated for each organ based on fluorescence intensity measurements using a Fluoroskan Ascent FL fluorescence plate reader (Thermo Labs) at 646-nm excitation/678-nm emission. The concentration of fluorescence reporter in the test samples were calculated on the basis of the standard curves.

Fluorescence scanning of whole mouse cryosections. Euthanized mice were frozen slowly in liquid nitrogen and stored at $-70{ }^{\circ} \mathrm{C}$. The frozen animals were embedded in a $2 \%$ carboxymethylcellulose (CMC) block, cut into $75-\mu \mathrm{m}$ sections and freeze dried for 12-16 h. The sections were scanned in fluorescence mode using a Fujifilm FLA5100 fluorescence scanner with a $635-\mathrm{nm}$ laser/665-nm long-pass filter.

In vivo animal imaging. An epifluorescence small-animal imaging system was employed, comprised of a 12-bit cooled charge-coupled device (CCD) camera, with a 660-nm laser source providing the excitation light. The animals were placed inside a box that prevents ambient light from disturbing the imaging procedure. Pharmacokinetic data was produced by acquiring one image frame every $30 \mathrm{~s}$ for $2 \mathrm{~h}$ (acquired with WASABI imaging software, Hamamatsu, Japan) from the time of contrast agent administration. For immobilization during the kinetics optical imaging procedure, the animals were anaesthetized in a coaxial open mask to light surgical level anesthesia with Isoflurane in a oxygen: nitrous oxide carrier gas. The animals were supplied with external heating from a heating blanket to sustain normal body temperature for the duration of the imaging. A Venflon catheter was placed into the tail vein for contrast agent administration. Each animal was given one contrast agent injection. The images were analyzed using custom-made MATLAB software (Mathworks), and were quantified in terms of raw target to background and signal to noise ratios and contrast enhancement dynamics. Image formation consists of subtraction of a calibrated camera bias from the raw CCD image data. The light source employed was inhomogeneous over the field of view, being of stronger illumination in the center of the image. In order to avoid illumination bias in the image a 'flat field' calibration of a uniformly reflecting object was used to normalize the fluorescence image. Washout kinetics for the contrast agent and negative control were estimated from the fluorescence time-intensity curves for regions of interest placed over the tumor and adjacent skin. The imaging time point for the targeting experiments was estimated as the time when the fluorescence signal from the contrast agent in the background or skin was approximately at the same level ( $\sim 2$ times) as the native fluorescence from the animal. Animals received the contrast agent (or negative control) intravenously at a dose of $50 \mathrm{nmol} \mathrm{kg}^{-1}$ body weight and were killed and imaged at the time point indicated from the kinetics measurement.

Competition. Excess of the unlabeled targeting peptide (180 or 650 times molar excess) was administered in a volume of $0.25 \mathrm{ml}$ i.v. $5 \mathrm{~min}$ before the i.v. administration of $50 \mathrm{nmol} \mathrm{kg}^{-1}$ body weight imaging dose of 
contrast agent in order to evaluate the effects of competition for the target. Experiments were performed in the high expression tumor type. Quantitative biodistribution was determined in blood, muscle, liver and tumor, as well as in vivo imaging in the mouse model mock-up of the clinical imaging conditions described below.

Mouse model mock-up of clinical imaging conditions. Imaging system. The imaging system used was built around a clinical laparoscope (model Hopkins II, S/N 26003 AA, Karl Storz, Germany). The laparoscope offers superior resolution to a fiberscope but was adjusted to produce the same spatial resolution as provided by a fiberscope. A $100-\mathrm{mW} 635-\mathrm{nm}$ diode laser was used as the excitation light source and fluorescence emission filters placed between the eyepiece and the CCD detector. The laser was mounted in the temperature stabilized laser mount and controlled by the laser driver and TEC (Thermoelectric cooling) controller. The laser light was passed through a mechanical shutter (Shutter controller: model SC10, ThorLabs; Shutter: model SH05, ThorLabsbefore entry into the liquid light guide (Light guide: model 495NB, Karl Storz, Germany). The fluorescence filters used were Omega Optical 3D filters with a long pass and band pass stacked together (660ALP/50 part no. 3012211 and 680AF40/50 part no. 3012209, Omega Optical, Brattleboro, VT). The CCD detector was a Hamamatsu ORCA-ERG (Hamamatsu Photonics, Japan), a progressive scan interline CCD with micro lens and IEEE 1394 FireWire interface. The effective number of pixels was $1344(\mathrm{H}) \times 1024(\mathrm{~V})$. The pixel clock rate was $14.75 \mathrm{MHz} /$ pixel with 16 frames per second at $2 \times 2$ binning. The r.m.s. readout noise is 8 electrons, a full well capacity of 18,000 electrons and 2,250:1 dynamic range. The chip can be cooled with forced air Peltier cooling to $-30{ }^{\circ} \mathrm{C}$. The dark current was 0.03 electrons/pixel/s. Readout was digitized to a nominal 12 bits. The quantum efficiency at $700 \mathrm{~nm}$ was $\sim 50 \%$.

Experimental procedure. The imaging probes were injected i.v. into the lateral tail vein and the animals were killed immediately before imaging. The optimal imaging time point was estimated previously from imaging pharmacokinetics studies (not reported here), with the same strain of mice. For each animal the subcutaneously grown tumors were excised post mortem. Tumor slices $1.6 \mathrm{~mm}$ thick and 3-4 $\mathrm{mm}$ in diameter were placed on top of a $2-\mathrm{cm}$ cleaned and longitudinally opened section of colon. The animal was then imaged with the imaging system.

Imaging system parameters. The spatial resolution of the images was set to correspond to a low spatial resolution similar to that afforded by a fiber-based implementation ( 32,000 pixels in the image field of view). A field of view of $4.44-\mathrm{cm}$ diameter was selected, giving an image area of $15.5 \mathrm{~cm}^{2}$. With 32,000 pixels covering the field of view, the pixel area is $0.0484 \mathrm{~mm}^{2}$ with linear pixel dimension (square pixel) of $220 \mu \mathrm{m}$. The distance of the object from the endoscope tip was adjusted (to $9 \mathrm{~cm}$ ) to provide the desired field of view from the laparoscope. The $-6 \mathrm{~dB}$ intensity level of the excitation laser light from the endoscope was used to define the dimension of the field of view. The camera binning was $2 \times 2$ with 0 on chip gain. The above settings (spatial resolution and field of view) correspond to those expected in a clinical system. An exposure time of $10 \mathrm{~s}$ was calculated to match the expected clinical illumination conditions.

Image artifact reduction. A piece of black card was placed directly above the kidney and bladder areas as necessary, to shield the kidney and/or bladder from excitation light and prevent artifact arising from the high fluorescence signal from the kidneys and bladder. This is not expected to be an issue in clinical imaging due to the greater distance separating tissues (in humans). Fecal material is also fluorescent. This is removed by flushing the opened section of colon. However, fecal material may be present in other parts of the intestine. This was confirmed by imaging with an excitation light wavelength that does not excite fluorescence from the dye, but still causes feces to fluoresce.

Target-to-background ratio. Two regions of interest (ROIs) covering the tumor slice and relevant colon background tissue were drawn manually (all regions defined by the same operator) with reference to the printed images annotated at the time of data acquisition. The mean and s.d. were calculated from pixels falling inside the region boundaries. Target-to-background ratios were calculated from these processed regions (mean signal from tumor ROI divided by mean signal from background ROI).
ROC analysis. A receiver operating characteristic (ROC) analysis was performed in order to quantify the efficacy of the compound in the animal model. This involves visually assessing images with the tumor both present and absent. The images are pre-processed before the visual assessment. It is important to use standardized image formation and presentation procedures for image interpretation. Standardized and automated image formation algorithms and presentation procedures were adopted.

Image pre-processing. There are four steps in the image formation process. 1 . Removal of camera bias by producing an image with the shutter closed with the same exposure time and read out rate used for animal image data collection. This background level is subtracted from the raw CCD camera data from the animal image. 2. Correction for illumination inhomogeneity: the illumination light is not homogenous, but it produces an illumination with a Gaussian-like profile. An image is acquired from a uniform target (stack of white paper), placed at the same distance from the laparoscope as the animal. This image is then used to normalize the fluorescence image in order to compensate for the illumination light inhomogeneity. The tumor piece is often placed centrally in the image, where the light is stronger (and hence the tumor will look relatively brighter). The normalization removes this bias. 3. Median filtering for noise reduction: the images contain noise. The noise content is dominated by photon (shot) noise. This arises due to the low light levels used to form the fluorescence image. A median filter with a $3 \times 3$ kernel is applied for noise reduction. 4 . Interpolation to reduce pixelation: the displayed image appears pixelated due to the relatively large pixel dimension. The pixelated appearance is reduced by $2 \times 2$ interpolation using a cubic b-spline interpolant.

Visual scoring. A standard visual scoring system employed for ROC analysis was used to assess the images. Assessors were required to score their assessment of the presence of a tumor section by giving a score from 1 to 5 where 1 = definitely absent, 2 = probably absent, $3=$ possibly present, $4=$ probably present and $5=$ definitely present.

There were two data sets for ROC analysis. Both data sets had a positive disease group (image of tumor slice placed on the colon), and a negative group (no tumor slice present). The first data set comprised of 18 animals given the imaging agent at a nominal dose of 1 nmole per animal and imaged 120 min after administration (positive group). The negative group had 16 images, with a total of 34 images in the first data group. The second data set was extended with additional animals that received a lower (one-third) dose, and animals imaged at additional time points as part of the dose and time point study. The second data set positive group $(n=39)$ was comprised of the 18 animals in the first data set positive group, plus additional images obtained from a dosing study ( 0.3 nmole per animal) and additional time point images $(60 \mathrm{~min}$ and $240 \mathrm{~min})$. The negative control group $(n=52)$ comprised the 16 images for the first group, plus additional images at the dose and times corresponding to the additional images from the positive group. Images obtained with the negative control substance (scrambled targeting sequence) were also included. There were 52 images in the negative group, giving a total of 91 images for the second data set.

Instructions to visual scorers. Prior to the visual scoring session the following instructions were provided for the scorers:

- There are 91 fluorescence images of open mouse colons to be scored.

- The extent of the opened colon section is given by the green line.

- The tumor is expected to provide positive contrast (appearing bright).

- There are one or zero tumor slices present in the image.

Images are from 1-nmole and 0.3 -nmole doses (50 and $17 \mathrm{nmol} \mathrm{kg}^{-1}$ body weight).

The standard scoring scale was presented, along with expected image examples (from a different image data set to that being scored), in order to indicate the expected range. In addition native fluorescence images acquired with green light excitation were also available in some instances where they identified obvious artifacts from fecal material. Scorers were blinded as to which treatment group the images belonged.

Image presentation. Images were presented in random order and scored by five observers who provided a consensus score. Images were also randomly flipped from left to right. 
Human subjects. Healthy volunteers were recruited from the CHDR (Centre for Human Drug Research) volunteer database. In addition, study-specific advertisements placed in local media and internet advertisements were used. 15 subjects at high risk of colorectal neoplasia were recruited by staff at the Gastrointestinal Department of Leiden University Medical Center. Subjects gave written acknowledgment of informed consent after receiving oral and written explanation of the study. A medical screening took place after they had consented to participate. After approval by the subjects, their general practitioners were notified when the subject was included in the study. The trial was registered in the EudraCT database (EudraCT number: 2010-019197-33). Please see Supplementary Methods for inclusion and exclusion criteria.

GE-137 safety testing in humans. The safety and tolerability of four ascending doses $\left(0.02,0.04,0.09\right.$, and $0.18 \mathrm{mg} \mathrm{kg}^{-1}$ of body weight) of GE-137 were evaluated in 20 healthy volunteers enrolled into 4 dose groups, each containing five subjects. In each group, four healthy volunteers received GE-137 injection and one received placebo $(\mathrm{NaCl} 0.9 \%)$ given intravenously by slow bolus injection. Healthy volunteers all underwent four sigmoidoscopy examinations without sedation after previous full bowel preparation, at 1,2, 3 and $4 \mathrm{~h}$ postinjection. A further 15 subjects at high risk of colorectal neoplasia received $0.13 \mathrm{mg}$ GE-137 per kg of body weight GE-137 and underwent a full colonoscopy $3 \mathrm{~h}$ after injection.

Human safety monitoring. At times indicated in Supplementary Table 4, venous blood samples and urine samples were obtained for clinical laboratory tests, for screening and safety monitoring purposes, and for antibody testing and pharmokinetic analyses. Samples were analyzed by the Central Clinical Laboratories of Leiden University Medical Center. The following assessments were performed: Hematology: hemoglobin, hematocrit, red cell count (RBC), MCV, MCH, MCHC, total white cell count (WBC), leukocyte differential count and platelet count. Biochemistry: sodium, potassium, calcium, chloride, total protein, albumin, glucose, urea, creatinine, creatinine phosphokinase (CPK), total bilirubin (total, conjugated), alkaline phosphatase, AST, ALT, gamma-GT and LDH. Coagulation: activated partial thromboplastin time and prothrombin time. Virology: hepatitis B, C and HIV serology (healthy volunteer study only). Urinalysis: leucocytes, blood, nitrite, protein, bilirubin, urobilinogen, $\mathrm{pH}$, specific gravity, ketone and glucose (Multistix $10 \mathrm{SG}$ (Bayer, Mijdrecht, the Netherlands) Urinary drug screen: cocaine, amphetamines, morphine, benzodiazepines, methadone and THC (healthy volunteers only). Urinary pregnancy test: CARDS O.S. H.C.G.-urine test kits (Pacific Biotech, Inc., San Diego, CA, USA). All female subjects underwent a pregnancy test unless they were surgically sterile or post-menopausal.

Additional safety measurements. Vital signs. Blood pressures and heart rate were measured using a Nihon-Kohden BSM-1101K monitor (Nihon-Kohden, Japan). Body temperature was measured tympanically with a BRAUN digital clinical thermometer (BRAUN Corporation, Germany).

Electrocardiogram. Standard 12-lead electrocardiographs were made using Nihon-Kohden Cardiofax (Nihon-Kohden, Tokyo, Japan). The following parameters were reported: PR-, QRS-, QT-, QTc- interval and heart rate. QT correction for heart rate was done using the Bazett formula. Continuous 1 lead ECG was performed from $5 \mathrm{~min}$ before IMP/placebo administration until $15 \mathrm{~min}$ after administration (using Nihon-Kohden BSM-1101K monitor, Tokyo, Japan).

GE-137 concentration measurement. Blood samples were collected using vacuum tubes containing sodium citrate as an anticoagulant (Becton Dickinson, NJ, USA). Samples were kept at $4{ }^{\circ} \mathrm{C}$, centrifuged within $2 \mathrm{~h}$ of collection (at $2,000 \mathrm{~g}$ at $4{ }^{\circ} \mathrm{C}$ for $10 \mathrm{~min}$ ), and plasma was split into two equal aliquots and stored at $-80^{\circ} \mathrm{C}$ until analysis. $100 \mu \mathrm{l}$ of plasma, $20 \mu \mathrm{l}$ of the internal standard (AH112959 (GE internal compound)) and $600 \mu \mathrm{l}$ of ice-cold precipitation solution (acetonitrile:water:CHAPS, (2:1:0.01, vol/vol/wt) were vortex-mixed for $30 \mathrm{~s}$ and then centrifuged for $15 \mathrm{~min}$ at $10,000 \mathrm{~g}$ at $6{ }^{\circ} \mathrm{C}$. The supernatants $(\sim 650 \mu \mathrm{l})$ were transferred to glass vials and dried under nitrogen. The dried extracts were dissolved in $100 \mu \mathrm{l}$ of $12.5 \mathrm{mM}$ ammonium acetate and $25 \mu \mathrm{l}$ from each sample was injected into a HPLC system (HP Agilent 1100 HPLC, CA, USA) and the compounds separated on an XBridge Shield R18 column, $3.5 \mu \mathrm{m}$ particle size (Waters, MA, USA), using a $25 \mathrm{mM}$ ammonium acetate/acetonitrile gradient run at $0.3 \mathrm{ml} \mathrm{min}^{-1}$. Column temperature was $35^{\circ} \mathrm{C}$. After separation, GE- 137 and the internal standard were detected by a UV-visible detector set at $650 \mathrm{~nm}$.

The calibration standards were prepared daily by diluting different amounts of standard solutions of GE-137 with human plasma in a range from 0.01 to $10.2 \mu \mathrm{g} \mathrm{GE}-137 \mathrm{ml}^{-1}$. Prior to analysis the calibration standards were spiked with the internal standard and treated similar to the human plasma samples. The calibration curves were made from the calibration standards by plotting the peak area ratio of GE-137 to the internal standard against the theoretical concentration of GE-137. The curve was fitted to a second order polynomial equation $y=a+b x+c x^{2}$ with a weighing factor $1 / y^{2}$. The amount of GE-137 in the samples was determined by calculating the same peak area ratio as above and correlating this ratio to $\mu \mathrm{g} / \mathrm{ml}$ plasma GE-137 using the calibration curve. Chromeleon version 6.6 (Dionex, CA, USA) was used for data sampling, integration, preparation of calibration curves and concentration determinations.

Colon wall fluorescence signal. From preclinical imaging studies, the optimal imaging time point was estimated to be approximately when the background fluorescence-imaging signal has cleared to one-half of its peak value. The background fluorescence washout time was calculated from serial measurement of colon wall fluorescence over time in healthy volunteers using two methods. In the contact method, the tip of the endoscope was placed gently on the surface of the colon wall, the endoscope light source was set to a standardized setting and eight images at non-overlapping locations in the distal part of the colon at each time point were recorded. In the reference standard method, a series of reference standards with calibrated fluorescence and optical properties were used. The reference standards consisted of titanium dioxide and iron oxide particles, to match the colon wall scattering and absorption coefficients respectively, and a small quantity of quantum dots for fluorescence, embedded in biocompatible epoxy resin. Reference standards inserted via the endoscope were temporarily held against the colon wall during imaging to provide a local fluorescence reference target. Again, eight images were recorded with the reference standard at different non-overlapping locations in the distal part of the colon at each time point. ROIs were positioned in the background tissue and the fluorescence reference standard for all snapshots at each time point. For each ROI, the mean and standard error of the fluorescence signal intensity in each pixel were calculated. Subsequently, mean and standard error were calculated and plotted at each time point. For contact measurements, the mean of the image signal intensity of the whole image was calculated. Subsequently, mean and standard error were calculated and plotted at each time point.

Optimal imaging dose and optimal imaging time point estimation. In the preclinical imaging studies the best achievable target-to-background ratio of a lesion was approximately 1.5:1. To achieve this ratio within a practical timeframe the optimal dose and optimal time interval between GE-137 administration and endoscopy were estimated to be $0.18 \mathrm{mg} \mathrm{kg}^{-1}$ ( = estimated clinical dose; $\mathrm{ECD}$ ) and $2 \mathrm{~h}$ respectively. (A higher dose will give a greater signal, but will require longer for wash-out from the background.)

To assess whether these results translated to humans, the fluorescent reference standards scaled to the dose-levels were used during the sigmoidoscopies conducted in the healthy volunteer. The reference standards provided a calibrated fluorescence target which could be used to compare relative fluorescence levels from adjacent structures and compare the fluorescence intensity to the preclinical models. The fluorescence levels were designed to provide a TBR of approximately 1:1 at one hour p.i. and corresponded to the expected fluorescence level in a lesion containing c-Met at 2 to $3 \mathrm{~h}$ after injection.

It was expected that the time taken for the reference standard TBR to change during sequential sigmoidoscopies from 1:1 to 1.5:1 would provide an estimate of the time needed to wait for sufficient background clearance for efficacy. This was approximately $2 \mathrm{~h} 40 \mathrm{~min}$ for dose groups $3\left(0.09 \mathrm{mg} \mathrm{kg}^{-1}\right)$ and $4\left(0.18 \mathrm{mg} \mathrm{kg}^{-1}\right)$. The imaging efficacy was deemed sub-optimal for the two lowest doses. The results from dose groups 3 and 4 were consistent with the contact measurement washout half-time, and indicated that optimal efficacy may be obtained within this dose range. 
It was agreed that the longest wait after injection that was practical for study logistics was $\sim 3 \mathrm{~h}$. The dose of $0.09 \mathrm{mg} \mathrm{kg}^{-1}$ showed optimal efficacy at $3 \mathrm{~h}$. It was expected that the colonoscopy exam in patients could take an hour. During this time the contrast agent would continue to wash out. To select the lowest possible dose while ensuring that good contrast was maintained for up to $1 \mathrm{~h}$ after the start of colonoscopy, a correction was applied to compensate for the washout. The washout was measured to be $10^{-0.12 \times \text { time }}$ and therefore the following correction was applied:

$$
\frac{0.5 \times \mathrm{ECD}}{10^{-0.12}} \approx 0.7 \times \mathrm{ECD}
$$

Therefore, an optimal imaging time of $3 \mathrm{~h}$ after injection, and an optimal imaging dose of 0.7 times ECD $\left(0.13 \mathrm{mg} \mathrm{kg}^{-1}\right)$ was recommended for use in the pilot study in patients.

Pilot study in patients at high risk of colorectal neoplasia. In 15 patients, a single i.v. dose of $0.13 \mathrm{mg} \mathrm{kg}^{-1} \mathrm{GE}-137$ was administered, based on the results of preliminary assessments in the healthy volunteers. Colonoscopy was performed at $3 \mathrm{~h}$ after injection. A single experienced endoscopist (J.C.H.H.) performed all the endoscopies. For the patient study the colonoscope was first inserted to the cecum using WL only. The colonoscope was then withdrawn while examining for possible neoplasia with each colonic segment being examined twice. The first-pass examination was performed using WL only. On detecting a lesion with WL, location in the colon, size, visibility in WL and the Paris classification were noted. Lesion visibility was classified as either 'visible lesion' or 'normal flat mucosa'. The lesion was then further examined for visibility in FL and its fluorescence characteristics were noted before biopsy. Fluorescence was related to background fluorescence and categorized as follows: clearly increased, mildly increased or same fluorescence as background. The endoscope was reinserted and a second-pass examination of the same colonic segment performed with combined FL and WL endoscopy. A large WL and smaller FL image were displayed simultaneously on the same monitor. Characteristics of lesions detected on the second pass were noted before biopsy.

Decisions regarding classifications in WL and FL were reached by consensus by the endoscopist and the endoscopy assistant during the procedure. As lesions could be visible in FL based on contours independent of their level of fluorescence, visibility of lesions in FL was assessed after the study day had taken place using the video images that were made during the procedure.

Lesion morphology was described according to the Paris classification and was based on the real-time imaging and snapshot data. Decisions on lesion classification based on review of the images were reached by consensus after assessment by two independent investigators (J.C.H.H. and A.M.J.L.). Size (in $\mathrm{mm}$ ) was estimated using open biopsy forceps $(8 \mathrm{~mm})$ held next to the lesion.

Fluorescence colonoscopy system. A commercial high-resolution video colonoscope was not available to provide simultaneous real-time imaging of WL and GE-137 emission fluorescence. The requirement of highly sensitive detection of fluorescence, as well as full flexibility of imaging parameters, necessitated the development of a custom instrument. To ensure patient safety, the instrument combines a custom image head mounted on the proximal end of a clinically approved lower GI fiber colonoscope (Pentax, FC-38LV) and medical grade components are incorporated where possible. The combined instrument has been tested for safety by an independent medical device testing and certification company (Intertek).

In addition to the commercial colonoscope, the system is comprised of an illumination module and an imaging head (Supplementary Fig. 6). The illumination module consists of a 150-W halogen lamp (Pentax LH-150PC) for WL and a high power (3-W) laser diode source (Modulight, ML1496) centered at $635 \pm 3 \mathrm{~nm}$ coupled into a $600-\mu \mathrm{m}$ diameter multimode fiber (Thorlabs, M29L01). The laser output power is restricted to Class 3B operation by limiting the light emitted from any accessible apertures to less than $500 \mathrm{~mW}$. The laser drive current is adjusted such that an irradiance of $\sim 10 \mathrm{~mW} \mathrm{~cm}{ }^{-2}$ is achieved at a working distance of $25 \mathrm{~mm}$ from the distal tip of the colonoscope.
The WL source and laser are filtered with a short-pass filter at $650 \mathrm{~nm}$ (Semrock FF01-650/SP-25) and a laser line excitation filter (Semrock, FF01628/40-25), respectively, before combining through a dichroic beam splitter (Omega Optical, 615DRSP.) The combined light is mated to the colonoscope illumination port through a custom mechanical adaptor and focused onto the ball lens of the port using an aspheric condenser lens (Thorlabs, AL2520-A).

The imaging head, mounted on the proximal end of the scope, consists of cameras, filters, and imaging optics. Light emerging from the proximal end of the colonoscope passes through a dichroic beamsplitter (Semrock, FF660Di02-25x36), separating the WL and fluorescence components into separate beam paths. The individual beams are imaged onto independent cameras using a 30-mm achromatic relay lens (Thorlabs, AC254-030-A1-ML) The lens focal length and diameter were chosen to minimize vignetting of the image.

To maximize sensitivity, fluorescence is filtered with an emission filter (Semrock FF01-692/40-25) to filter out wavelengths outside 672-712 nm and detected with a monochrome Peltier-cooled (to $-20^{\circ} \mathrm{C}$ ) electron multiplying charge coupled device (EMCCD, Retriever Technology RT-XMV-247.) The camera pixel count is $658(\mathrm{H})$ by $496(\mathrm{~V})$, running at eight frames per second (8 f.p.s.) and a 94-ms exposure time. For WL video, a color camera is used (Sentech, STC-CLC152A) with 1392(H) by $1040(\mathrm{~V})$ pixels running at $8 \mathrm{fps}$ and a 40-ms exposure time. To avoid excessive leakage of the red excitation laser into the WL channel, a laser rejection (notch) filter (Semrock, NF03$633 \mathrm{E}-25)$ is placed in front of the color camera. The entire imaging head is mounted in a metal housing (Rittal, JB080804HC), which serves as a heat sink for passive cooling of the cameras. The metal housing is attached to the medical cart (Endocart, ED-100B) via an articulated support arm (Bretford, FPSMWADJ2XXAL) that ergonomically supports the weight of the imaging head yet provides six degrees of freedom for the required range of motion.

Images are acquired via a single-board, dual-channel frame grabber with CameraLink interface (Matrox Imaging, Solios eCL SOL-6M-CL-E), using custom image-acquisition software programmed in Microsoft Visual Studio 2005 and Matrox Imaging Library (MIL) version 9.0. The computer system consists of a workstation (Hewlett Packard, xw8600) running 32-bit Windows $\mathrm{XP}$ and two 24-inch monitors (Hewlett Packard, LP2475w) one of which is mounted on a support arm (Endocart, EDS-305) and the other on a satellite medical cart (Endocart, EMS-104).

The raw video streams are directly recorded to RAID0 drives in AVI file format. During live image acquisition, video streams from both channels are digitally filtered with a finite impulse response (FIR) filter to remove the fiber honeycomb pattern. FIR kernel sizes up to $9 \times 9$ pixels may be implemented without appreciable delays on display and recording frame rates. Following each procedure, data is archived to a $1 \mathrm{~TB}$ external drive (Western Digital WDE1UBK10000N). Electrical isolation of the entire imaging system is achieved with a medical-grade isolation transformer (Tripp Lite IS1800HG) with up to an 1800-W capacity (Supplementary Fig. 6).

Histopathology. All biopsies were formalin fixed, paraffin embedded, sectioned, and stained with $\mathrm{H} \& \mathrm{E}$ according to the standard protocol of Leiden University Medical Center. Final histological evaluation was performed by a single specialist GI pathologist (H.M.). For further analysis all diagnoses were grouped into three basic categories according to the WHO classification of tumors of the digestive system: normal, hyperplastic polyps and adenomatous polyps.

Immunohistochemical analysis of c-Met expression. $4-\mu \mathrm{m}$ sections were deparaffinized, rehydrated and heat treated $\left(100^{\circ} \mathrm{C}\right)$ in Dako Cytomation Retrieval Buffer pH 9.0 (Dako, Glostrup, Denmark) for 12 min using the Biogenex microwave EZ Retriever (Biogenex, Fremont, CA). Slides were then immersed in 3\% hydrogen peroxide solution for $10 \mathrm{~min}$, blocked with Serum-Free Protein Block for 20 min and then incubated for 60 min with a primary rabbit monoclonal antibody specific for the $\mathrm{N}$ terminus of human c-Met (antibody EP1454Y, Abcam, Cambridge, UK) at room temperature at a concentration of 1:150 using the i6000 Infinity automated staining system (Biogenex, Fremont, CA). Slides were subsequently incubated with HRPconjugated goat anti-rabbit IgG for $30 \mathrm{~min}$ at room temperature, antibody binding was visualized with diaminobenzidine (Dako) and slides were 
counterstained with hematoxylin. Positive control sections known to be c-Met positive and a buffer negative staining control in which the primary antibody was omitted and replaced with the primary antibody diluent were included for each staining run.

Analysis of slides to ascertain the extent and intensity of c-Met staining took place at Pathology Diagnostics Ltd., Cambridge, UK. A board-certified anatomic pathologist determined the score for each section by visual assessment of 10 fields of view (FOV) at $40 \times$ magnification using light microscopy. All stained slides had been relabeled and were assessed in a blinded fashion. The c-Met staining intensity of the epithelial cell membrane was evaluated for each FOV using a four-point scale of 0 (no staining), 1 (weak staining), 2 (moderate staining) and 3 (strong staining). The percentage area occupied by epithelial cells was determined for each FOV by image analysis using Image Pro Plus software (Media Cybernetics, US). The percentage area occupied by epithelial cells per FOV was multiplied by the epithelial cell membrane staining intensity in the same FOV to reach a score $(0-300$ arbitrary units) for each FOV. The final score for each slide was the calculated mean for the $10 \mathrm{FOV}$.

Fluorescence immunohistochemistry. $4-\mu \mathrm{m}$ sections were deparaffinized, rehydrated and incubated overnight at $4{ }^{\circ} \mathrm{C}$ with c-Met monoclonal rabbit primary antibody (Abcam, EP1454Y, ab51067) diluted 1:100 in PBS. Sections were then in incubated with Alexa Fluor 488-goat anti-rabbit IgG (Invitrogen) diluted 1:100 PBS for $1 \mathrm{~h}$ at room temperature and mounted with SlowFade Gold Antifade Reagent with DAPI (Invitrogen). Sections were analyzed with a Leica DM5500 B fluorescence microscope (Leica Microsystems) using Cy5, FITC and DAPI filter settings and dual-color overlay images were made using ImageJ software. Control sections in which the primary antibody was omitted were performed with each separate experiment. Control images were taken using identical settings to those used for sections where primary antibodies were used.

Ex-vivo fluorescence measurements of tissue blocks. Fluorescent signals in formalin-fixed, paraffin-embedded tissue blocks each containing a single biopsy taken from endoscopically normal colon or lesions seen at colonoscopy from the patient study were measured for all specimens using an IVIS Spectrum scanner (Xenogen, San Francisco, CA, USA) as we have performed previously ${ }^{32}$. Tissue blocks were analyzed using Cy.5 settings (excitation $640 \mathrm{~nm}$, emission $680 \mathrm{~nm}$ ). Fluorescence intensities (photons $\mathrm{sec}^{-1} \mathrm{~cm}^{-2}$ ) were calculated using Living Image software (Xenogen). The target-to-background ratio was calculated by dividing the fluorescent intensity in tissue samples from lesions with abnormal histology (adenomatous or hyperplastic) by the fluorescence intensity in healthy control tissue samples of the same patient.

Statistical analyses. A contingency table for the histology groups and the categories of fluorescence was analyzed for association with a Fisher's exact test. As the 130 biopsies were analyzed from 15 patients and most patients had biopsies with a fluorescence level in all three fluorescence categories and a histological diagnosis in all (grouped) categories, within subject comparisons seemed appropriate for the analysis of the association between c-Met expression and fluorescence levels and c-Met expression and histological diagnosis. The analysis was performed with a mixed model analysis of variance with 'fixed factor' fluorescence category or grouped diagnosis and 'random factor' subject with a variance components covariance structure. Averaging the c-Met expression within subject and category was not necessary for the analysis to be feasible. The following 'contrasts' were calculated within the model: mildly increased fluorescence versus the same fluorescence as background; clearly increased fluorescence versus the same fluorescence as background; clearly increased fluorescence versus mildly increased fluorescence. The analysis was repeated with 'fixed factor' grouped diagnosis and the following 'contrasts' were calculated: hyperplastic polyp versus normal; adenomatous lesion versus normal; hyperplastic polyp versus adenomatous lesion. Data were shown as least-square means with $95 \%$ confidence intervals as error bars. All calculations were performed using SAS for windows V9.1.3 (SAS Institute, Inc., Cary, NC, USA).

Ethics. The protocol of the studies in humans was approved by the Medical Ethics Committee of Leiden University Medical Center. The CCMO (Central Committee on Research involving Human Subjects) acted as the Competent Authority. The study was conducted according to the Dutch Act on Medical Research involving Human Subjects (WMO).

Additional methods. Detailed methodology is described in the Supplementary Methods.

32. van der Poel, H.G., Buckle, T., Brouwer, O.R., Valdes Olmos, R.A. \& van Leeuwen, F.W Intraoperative laparoscopic fluorescence guidance to the sentinel lymph node in prostate cancer patients: clinical proof of concept of an integrated functional imaging approach using a multimodal tracer. Eur. Urol. 60, 826-833 (2011). 